\title{
THE CONTRIBUTION OF CLIMATE SERVICES TO SUSTAINABLE WINE PRODUCTION IN SAN JUAN, ARGENTINA
}

\author{
ZUZANA BOUKALOVÁ ${ }^{1}$, JAN TĚŠITEL $^{2}$ \& ZBYNEK KŘIVÁNEK ${ }^{3}$ \\ ${ }^{1}$ VODNÍ ZDROJE, a. s., Czech Republic \\ ${ }^{2}$ METCENAS o. p. s., Czech Republic \\ ${ }^{3}$ Czech Center for Science and Society, Czech Republic
}

\begin{abstract}
This paper deals with a description of the technical components of the system ALICE and their practical application under the project of the EUREKA programme: "ALert system In Case of Excess withdrawal or rise up of groundwater in the sensitive areas" (ALICE) in San Juan, Argentina. The ALICE system is used for sustainable wine production to achieve an optimum yield (in quantity and quality) and farm income with a minimum input (nutrients, water, but also energy, pesticides, herbicides, money), whilst preserving the environment. The system works by recording signals from a number of points of measurement - sensors located within the site of interest, and further by transmitting these signals by radio to a pre-processing node and subsequently, over the Internet, to a central database. In this paper we are discussing how the assignment carried out in Argentina was specified and how an analysis was made of the system possibilities in the catchment scale, with regard to required performance, users, and necessary operations, monitoring methods, and required resources.
\end{abstract}

Keywords: climate services, water management, sensors, sustainable irrigation, alert system, Argentina, wine farms.

\section{INTRODUCTION}

Climate services are becoming part of everyday life of a great number of end users from various branches, providing climate information in different contexts in ways that enhance decision-making of individuals as well as organizations. At the present time, climate services are beginning to be used by different end user groups having specific needs and operating conditions influenced by climate change. The climate service market has only started to develop and the end users' interests in information, the way it is used and obtained, are being identified. At the same time, the awareness of potential target groups about the possibilities concerning climate services, their prices and the effectiveness of their use is being raised and efforts are made to strengthen the confidence of end users in these new services. Climate services can be very effectively used for tackling possible problems and conflicts of interest within the river basin, either separately in different management areas (such as water supply, agriculture, ecosystem and environmental impacts, pest \& disease control, river system risk analysis, etc.), or in an integrated form, which means that the interdependence of selected climate services on a river basin scale is investigated (e.g. the interconnectedness of climate services for river system risk analysis and agriculture/crop management).

The project ALICE ("ALert system In Case of Excess withdrawal or rise up of groundwater in the sensitive areas"), that was finished in December 2016, clearly contributed to the use of climate services in water management and precision farming. ALICE dealt with developing a new generation warning and monitoring system based on the implementation of interoperable interfaces that enable early spotting of extreme drops or rises of groundwater levels in areas that are directly dependent on their stability or informing about the soil moisture to optimize irrigation. 
The testing of the ALICE system was conducted at the vineyard of El Huaico in Valle Pedernal, province of San Juan, Argentina, where the own WSN (Wireless Sensor Network) system has been designed.

What makes this site interesting is that the project aims to develop the existing and create new climate service markets, and especially to reinforce and expand the climate services to new users using the pilot's application and best practices. Even though the ALICE system has been tested at one vineyard only, it can be, after successful calibration, used at a subcatchment to catchment scale for instances when optimal management of water use by various end users is needed, be it as part of agriculture - viniculture (such as at another potential site of Valle Tulum, Argentina, where irrigation is done through three canals from one source), or by end users from various coexisting areas (such as agriculture, water management, industry, etc., as is the case in the catchment of Litovelské Pomoraví, CR). Thus, the ALICE system can be an important technological feature while implementing a more practical approach to water management at a catchment or sub-catchment scale where water resources are under big pressure and water uses need to be optimized given the limited resources and risk management issues within the river basin.

\section{ALICE METHODOLOGY}

The pilot site in Argentina, San Juan was the farm of El Huaico which covers an area of approx. 354 ha (of which 80 ha are currently productive vineyards where the Malbec variety has been grown since 2008) in the southern part of the Pedernal valley. In the pilot area, soils are alluvial, made of gravel of varying granulometry combined with sands, silts and clays. The proportions of the materials vary depending on the place. The soils have different physical properties as well as different water retention capacity and generally, the soils are poor showing low content of organic matter. As for climatic conditions in the area, the highest average temperatures range between $28^{\circ}$ and $24^{\circ} \mathrm{C}$ in the period of grape ripening from January to April, while the lowest average temperatures range between $10^{\circ}$ and $4^{\circ} \mathrm{C}$. The average annual precipitation - despite being higher than in the other valleys within the province - is low, reaching only a little more than $150 \mathrm{~mm}$. In the area of interest, water for irrigation is extracted exclusively from groundwater sources by means of wells [1].

The pilot installation of the ALICE system technical equipment in the vineyard of El Huaico was carried out in two stages (Stage I: September 2015, Stage II: January/February 2016) and followed the hydrogeological and pedological surveys of the site in March 2015. [2]. During the survey, it had been determined that in some parts of the vineyard the vine did not reach the required height, the plants being considerably lower and creating little islands in the middle of more vigorous vines. In other parts of the vineyard, in turn, there are overgrown plants which seem to be very good quality and resilient but do not bear grapes. Both of these extreme situations concerning good vine growth at the site are considered serious, even critical, by the vineyard management. Therefore, it is necessary to eliminate such differences in vine growth by a change in management methods in the future. The most likely cause of the uneven vine growth in the Pedernal valley was found to be the spatially uneven water distribution which is likely to be caused by hydrogeological conditions of the zone as well as the distribution of preferential pathways of ground and subsurface water. The excessive growth of some other plants can be accounted for by an oversupply of nutrients as well as other factors [3].

ALICE pilot plant (Fig. 1) based on Wireless Sensor Network (WSN) system was installed in situ at the vineyard of El Huaico pilot at Pedernal and became fully operational from February 2016. 

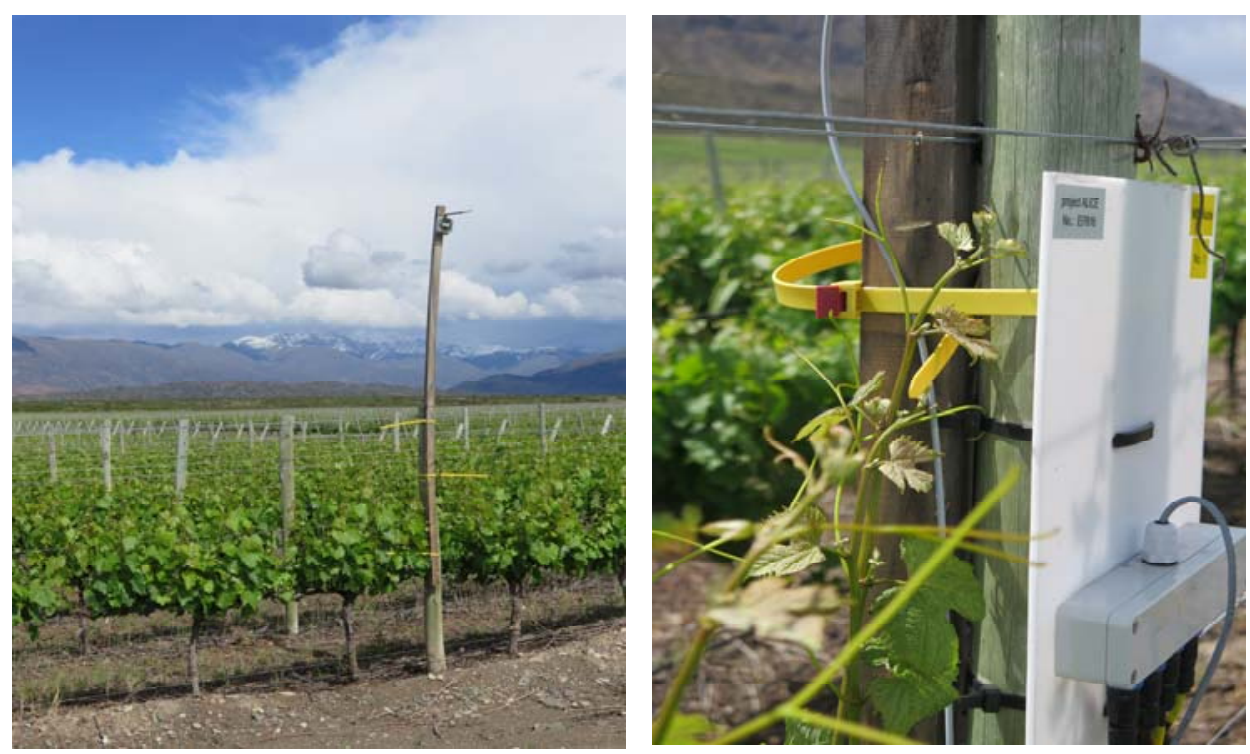

Figure 1: ALICE pilot plant in valley Pedernal.

The WSN system is based on the sensors that are monitoring soil moisture and temperature; these sensors were placed in the different sectors of the vineyard (to the three different depths of $20 \mathrm{~cm} / 60 \mathrm{~cm} / 110 \mathrm{~cm}$ below the terrain) to obtain the information from the sectors with different modus of the wine growth [3].

Next, ALICE project simulated the pilot of the climate services in agriculture, where meteorological and sensor data, partly as well as satellite data, are used for the optimization of wine growing in the valley Pedernal. In the San Juan agricultural area, the one of the basic prerequisites for a successful crop is keeping the optimal irrigation pattern, specifically the irrigation (fertilization) efficiency control on vineyards using on-line sensing systems providing information on soil moisture and temperature and other parameters, if needed.

At valley Pedernal, the use of climate services by target group in a vineyard sector had been defined as a model proceeding that can be transferred to other similar sites or generally promoted from a local to a regional level in agriculture and water management.

\section{HARDWARE SOLUTION - SYSTEM DESCRIPTION}

The ALICE system works by recording signals from measuring points - sensors placed in pits or boreholes at the area of interest. These signals are radio-transmitted to a pre-processing unit and further transferred through the Internet to a central database accessible to authorized persons only. The used measurement technology is sufficiently universal so that it can be conveniently adapted. The system measures previously defined parameters (based on the end users' requirements as well as local or regional conditions), continuously storing the data on a server and making them accessible to the users (their software systems) through web services. At present, interoperability is vital. Therefore, the data are best provided in a format that is readable by as many clients as possible.

For the application on the pilot site, the ALICE WRS system hardware configuration is composed of: 
- Communication gateway that contains several modules, some of which are optional:

- Power supply of $3.3 \mathrm{~V}, 5 \mathrm{~V}$ for the system

- Processor - control processor STM32F207

- GPRS - GPRS wireless network module, or

- Iridium - satellite wireless network module Iridium SBD

- IQRF - data concentrator unit for the wireless sensor network (node 0)

- optional backup lead-acid battery

- antenna for Iridium IRD-SAF5250

- cable RG58 10m

- 868MHz whip antenna

- serial extension cable

- Sensor node

- $868 \mathrm{MHz}$ antenna

- Optional sensors with SDI-12 interface

Table 1: Technical specifications of the gateway that is used as a data concentrator, transmits measurement requirements, receives the data, stores them in a readonly memory and sends data to the server.

\begin{tabular}{|l|l|}
\hline Supply voltage & $=12-32 \mathrm{~V}$ \\
\hline Power consumption & Max. of $2 \mathrm{~A}$ at $12 \mathrm{~V}$, typically $200 \mathrm{~mA}$ at $12 \mathrm{~V}$ \\
\hline Temperature range & -20 to $+70{ }^{\circ} \mathrm{C}$ \\
\hline Dimensions & $180 \times 130 \times 110 \mathrm{~mm}$, connectors stick out \\
\hline Material of the cover & ABS \\
\hline Protection & IP66 \\
\hline Connectors & $2 \times$ SMA, power supply cable entry, debug \\
\hline
\end{tabular}
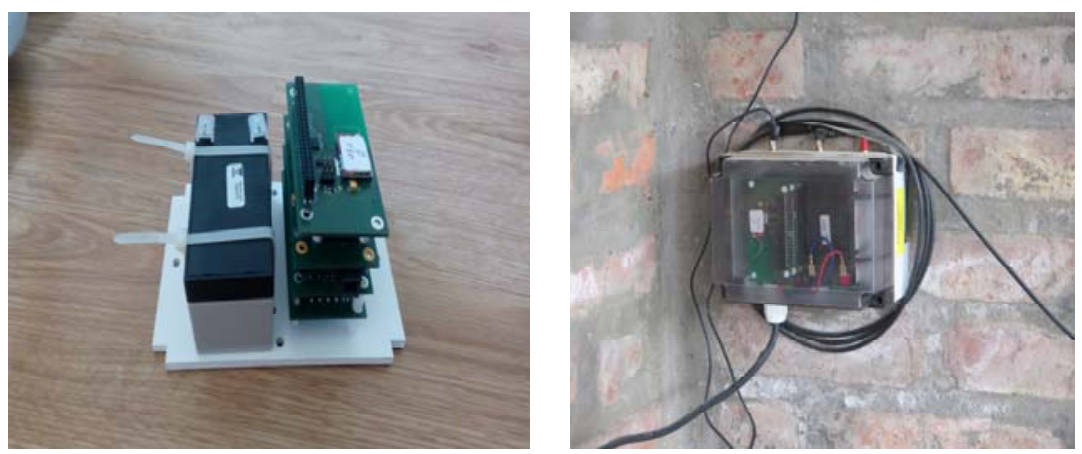

Figure 2: The gateway in situ - valley Pedernal.

Table 2: Technical specification of the sensor node.

\begin{tabular}{|l|l|}
\hline Power supply & C type lithium battery of 3.6V \\
\hline Power consumption & $250 \mathrm{uA}$ sleep, 19mA transmission \\
\hline Transmission band & $868 \mathrm{MHz}$ \\
\hline Maximum emitted radio power & $12.5 \mathrm{~mW}$ \\
\hline Temperature range & -20 to $+70^{\circ} \mathrm{C}$ \\
\hline Dimensions & $80 \times 82 \times 55 \mathrm{~mm}$ \\
\hline Protection & IP66 \\
\hline
\end{tabular}


A sensor node is powered by a primary $3.6 \mathrm{~V} \mathrm{C}$ size lithium battery with a 1-year lifetime (1 season). If the node is also used for retransmission of data from another node or more frequent measurements are required (in the model case, 2 daily measurements are taken), or the node works at low temperatures, battery lifetime is shorter. During pilot application, we found out that for the proper functioning of the system, the node must be positioned as close as possible within cable reach of the respective sensors (approx. $3 \mathrm{~m}$ ) and the cable itself must not form large loops that may lead to the induction of dangerous voltage levels. To ensure maximum radio range of the node, at least the node antenna must be placed vertically at a minimum height of $1 \mathrm{~m}$ above the surrounding canopy. Each node can collect data and, at the same time, works as a router - meaning that it can transmit data from other nodes (flooding method). If a node that receives or sends data has no coordinator within range, it will transmit the data to a router in the direction of the coordinator. The coordinator receives the data and retransmits them to a router that can finally reach the coordinator.

In designing the distribution of nodes, we took into consideration especially the node's radio range, which about $500 \mathrm{~m}$ is provided if the installation instructions are followed (the range being limited by regulations concerning the radiated power for the given band, the antennas used, etc.). Each node must have at least one other node or directly a network coordinator in its vicinity. However, for network robustness it is preferable to have more nodes within reach so that the radio pathway is backed up in case of a router node failure. If it is not possible to reach the coordinator point using only measurement nodes, additional nodes without sensors may be placed that will work merely as routers.

In the ALICE system, only sensors with SDI12 digital interface can be connected to a measurement node, as the node supplies $12 \mathrm{~V}$ power that is switched on for the measurement period only. Given the limited time for measurement, some sensors may not work properly.

Sensors certified by the manufacturer that have been used in the ALICE pilot application in the Pedernal valley:

- Decagon MPS6 - Water Potential

- Decagon VP3 (VP4) - Relative Humidity, Barometric Pressure, Air Temperature, and Vapor Pressure

- Decagon 5TM - Soil Moisture \& Temperature Sensor

- Decagon 5TE - Soil Moisture, Temperature, \& Electrical Conductivity Sensor

- Campbell Scientific CS650 - Water Content Reflectometer.

For data reception, a server with a public IP address needs to be used on the Internet, on which an application (daemon) runs for reception and possible storage, presentation, processing, etc. The application must be able to receive "sensob" protocol data if GPRS data channel is used. For the ALICE system application, the receiving daemon runs at sensors.lesprojekt.cz at port $4252 \mathrm{UDP}$.

In case of the Iridium data channel, the "sensob" protocol must be used that has an optimized form for data volume transmission - the modem uses the SBD (Short Burst Data) transmission that is incompatible with the "sensob" protocol. For the ALICE system application, the receiving daemon runs at sensors.lesprojekt.cz at port 4254 TCP.

After the reception of data, they are re-confirmed and deleted from the operational data store (internal flash memory). Then they are stored in a database (postgres).

The ALICE system pilot installation in Argentina at the site of El Huaico, Pedernal Valley, has been used to develop the utility model "Configuration of an optimized system for irrigation control support using a large wireless data collection network" that was registered at the Industrial Property Office of the Czech Republic under No. 2016-32877 on $29^{\text {th }}$ November 2016. 


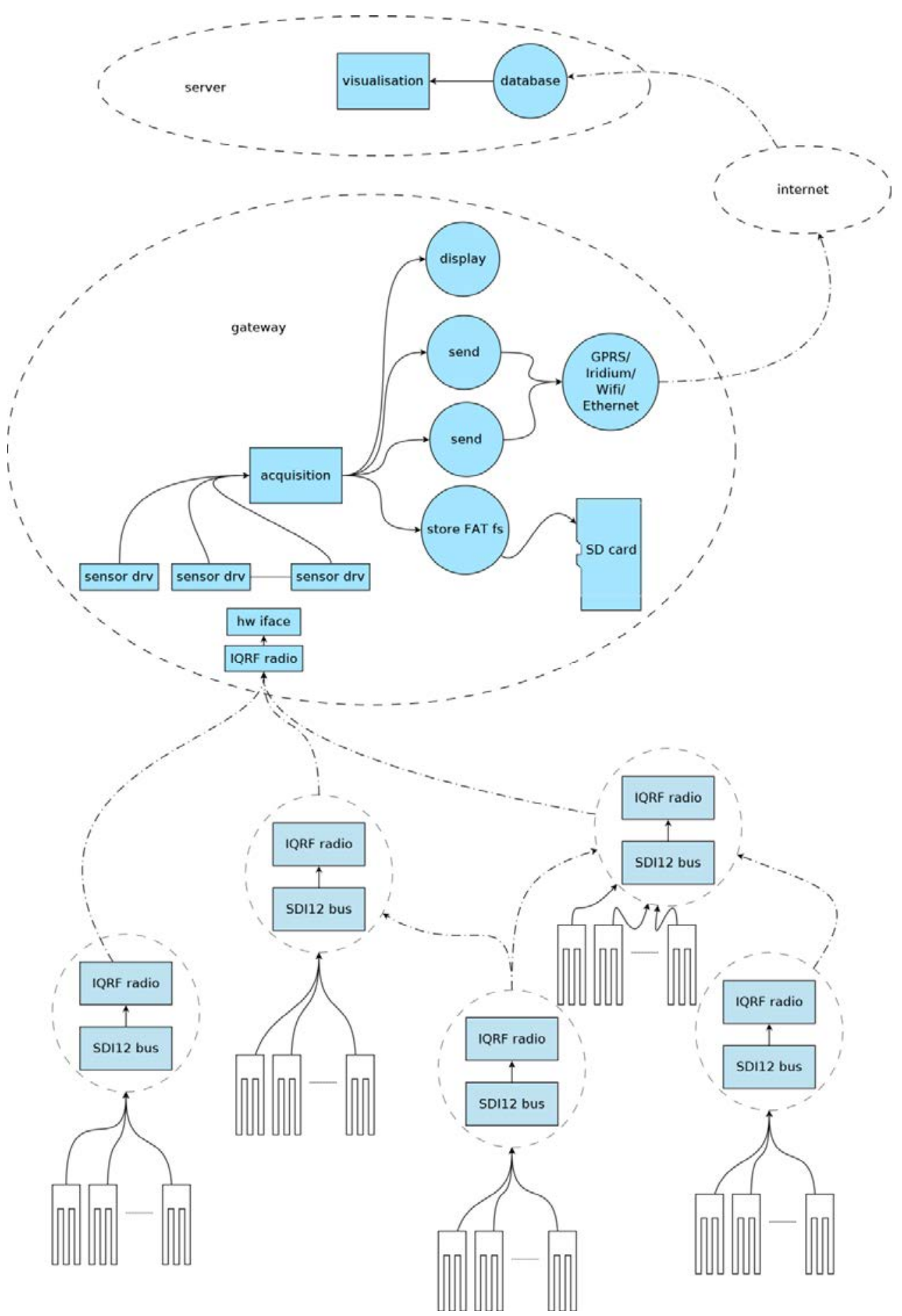

Figure 3: ALICE WRS and how is functioning. 


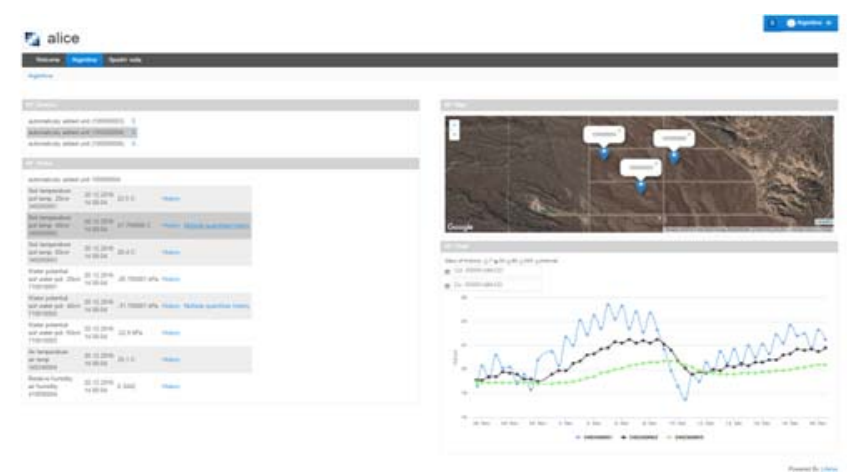

Figure 4: Screen with a graph for a selected parameter from all sensors of the respective measurement node (for a selected period).

\section{POTENTIAL CLIENTS FOR THE ALICE SYSTEM AND ITS POSSIBLE PRACTICAL APPLICATIONS}

As mentioned in Section 1 (INTRODUCTION), the testing of the ALICE system was done at the vineyard of El Huaico in the Pedernal Valley, province of San Juan, Argentina. This vineyard is one of the many owned by the company Bodegas Callia [4] which is a huge wineproducing company with Dutch owners. This company was chosen for the testing of the system based on the assumption (justified in theory and many times confirmed in practice) that bigger enterprises are more willing to implement new technologies, another reason being that the initial investment in the installation of the system and its subsequent operation costs would represent only a minute fraction of the company's total budget.

Looking back on the two-year experience we have with this company; however, we have to admit that this choice was not the best we could make for two reasons.

Firstly, the company management is detached from the ownership of the business. This fact was not fully appreciated; as a consequence, complications arose in communicating with the company's representative (vineyard manager). The vineyard manager is an employee, not the owner of the vineyard. As a result, his room for manoeuvre is considerably limited by the fact that he is hired by the owners and he takes pains to avoid any risk that could possibly threaten his position.

Secondly, the Pedernal Valley has no Internet coverage, which in selecting the ALICE project pilot site was originally regarded as a positive challenge by us. Due to the lack of coverage, the processed information was not accessible on-line at the vineyard (the implemented Internet connection via satellite was just one-way for price reasons). Instead, it could be accessed from an office in San Juan (at a two-hour ride from the pilot site) or from other places where direct connection to the Internet for the evaluation of data on the server was possible. This, however, lacked the required "immediate" effect as far as the on-site irrigation optimization was concerned.

Based on this experience, it is obvious that the target group for another transfer of the system should be defined by at least two criteria:

- The potentially addressed clients should be the owners of the vineyard where the system is to be operated.

- The vineyard should have Internet coverage to ensure smooth/affordable transmission of data and their immediate utilization. 
In performing an analysis of the competitive environment, we did not identify any system that would offer the same (or at least similar) services. The only data gathered by some winegrowers are meteorological data from small weather stations located on their vineyards.

In the province of San Juan, there is considerable interest in the services provided by the ALICE system, as we found out in a field survey. Based on the respondents' opinions, we assume there is similar interest in the province of Mendoza. In considering the implementation of the system, cost-effectiveness is regarded important by potential users. As one of the INTA (Instituto Nacional de Tecnología Agropecuaria - a public institution that has been established in Argentina for 60 years now, providing consulting services for the agricultural sector with a view to encourage rural development) consultants pointed out in discussing the matter with us, "wine-growers will be reluctant to implement a technology that will bring them no immediate profit".

The price of the system itself could be determined by us after potential site and end-user's conditions monitoring and evaluation. The price respects the modular structure of the system, which means that each module is priced separately. However, for the evaluation of the ALICE system performance (in terms of irrigation water saving, yield increase or yield stability over time...), a longer operation period is necessary clearly exceeding the scope of the project as well as the demonstration possibilities of the pilot installation (run in the Pedernal Valley from February 2016 to December 2016).

The "snowball method" based on the promotion of good examples (exempla trahunt) was evaluated by us as the most effective strategy for market uptake and further spread of the ALICE system as part of the agricultural climate services. In this regard, we managed to identify potential interested entities in the province of San Juan in cooperation with the PROSAP [5] programme representatives.

\section{CONCLUSION}

Generally, the ALICE system is developed for geographical areas / catchments where the stability of groundwater levels is a vital precondition for their agricultural use, and for settings where groundwater level can be controlled. However, ALICE could be used as well for the irrigation optimization in any other case (like Pedernal valley, where the groundwater is $80 \mathrm{~m}$ below the terrain). The challenge for sustainable wine crop production in Pedernal, el Huaico farm, is to achieve optimized yield (in quantity and quality) and farm income with a minimum of inputs (nutrients, water, but also energy, pesticides, herbicides, money), while preserving the environment.

In the province of San Juan, Argentina, the ALICE system represents an unrivalled solution that has no parallel in the area. Especially small wine growers and farmers show considerable interest in the climate services provided by the ALICE system in the field of viniculture. In the provinces of San Juan and Mendoza, practical application of the ALICE system on a larger scale and its market uptake is based on at least two conditions. The first is not only a successful on-site installation and operation of the system but also their proper promotion (exempla trahunt). The other condition is potential public support provided by the state (province) in the form of a "new subsidy scheme" within the running PROSAP programme.

As in the previous case, in negotiating the introduction of a new aid scheme, the activity of the local coordinator, who is indispensable for the spread of the new technology among end users, is vital. As a matter of fact, entities potentially interested in applying the ALICE system can be identified in other agricultural branches than that of viniculture in Argentina. 

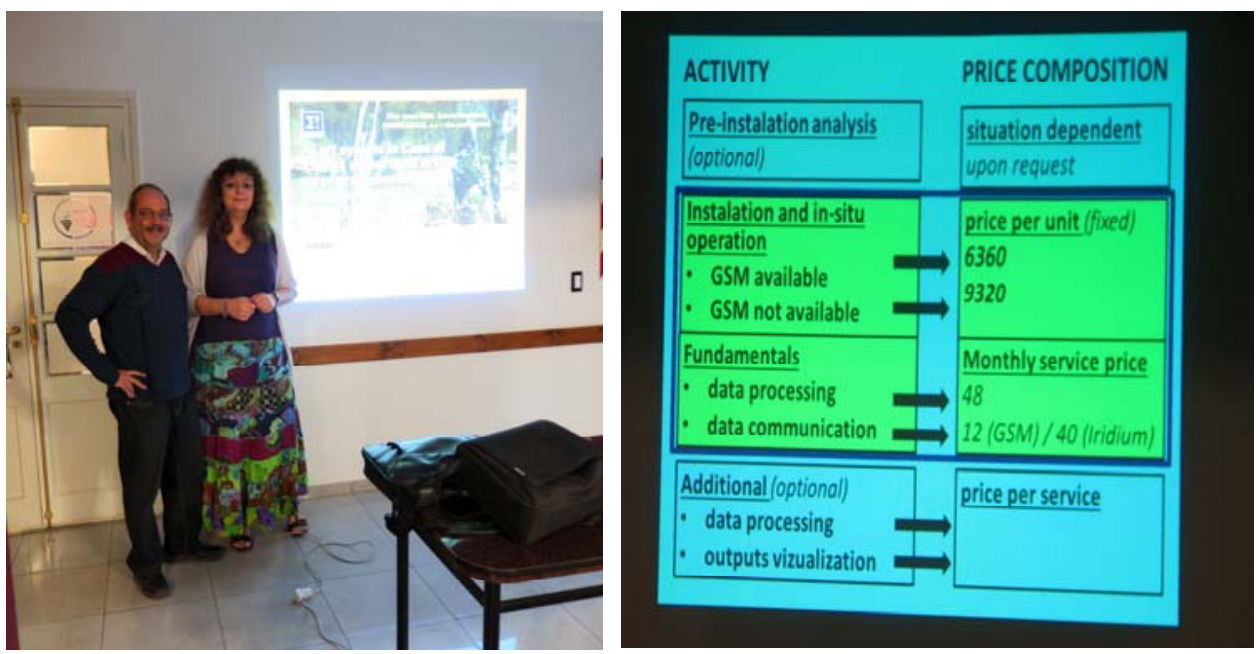

Figure 5: Training for the ALICE system application in agricultural climate services at the headquarters of Federacion de viñateros y productores agropecuarios de San Juan on $23^{\text {rd }}$ November 2016. Leaders: ALICE coordinator (Z. Boukalova), Local coordinator (O. R. Dölling).
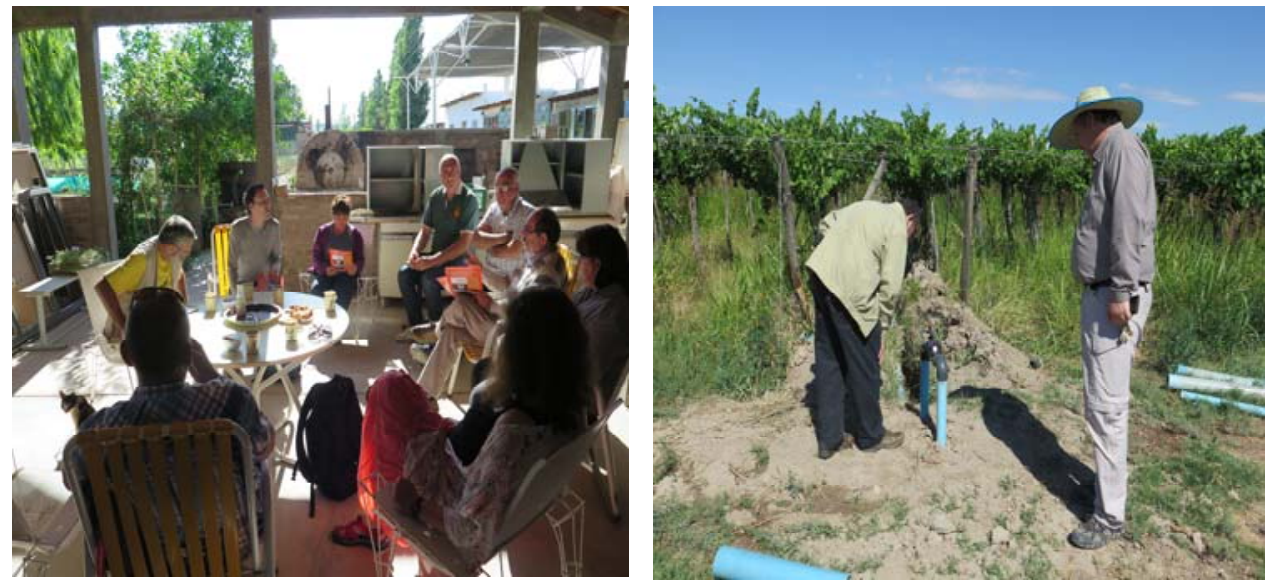

Figure 6: Negotiations with potential ALICE system end users at the farm of Crescentino Hermanos - Caucete and Conte Grand.

The ALICE system may be adapted to different hydrogeological and climate conditions, such as for Europe, in the river basin management. The application of the adapted system is possible not only as part of agricultural climate services but in other sectors as well, such as in water management and on-line monitoring of groundwater levels including the evaluation of their fluctuations over time with a view of resolving the related conflict situations among various potential groundwater users. 
A good example of how the already tested ALICE system can be used in river basin management is its implementation in an area of riparian forests and wetlands (the Litovelské Pomoraví catchment), which is also used as a source area for drinking water extraction. Generally, the amounts of groundwater extracted for agricultural and industrial purposes are increasing in the area as well. The quality and the very existence of the ecosystems in the catchment area depend directly on the stability of groundwater levels. If an extreme decline in groundwater level takes place, permanent damage to these ecosystems may result. When the groundwater level declines beyond the critical limit, the root systems of the riparian forests or wetlands will lose access to water and the decline of the (often protected) ecosystems along with irreversible biotope degradation will take place. This, in turn, may impact the quality and quantity of the water resources within the catchment. In the future, the ALICE early warning system integrated into the river basin management will very effectively serve for active protection of the protected areas of riparian forests and wetlands, at the same time enabling maximum groundwater extraction for drinking and industrial purposes. As a building block of the river basin management system, however, it can as well be used to address other water management issues and conflicts of interest than those mentioned above. The ALICE system is ready for immediate application in water use optimization on a river basin scale.

\section{ACKNOWLEDGEMENTS}

This paper was developed thanks to the E!7616 EUREKA project ALICE, co-financed by the Ministry of Education, Young and Sports, Czech Republic (50\%) and organizations VODNÍ ZDROJE A. S. and Czech Center for Science and Society (50\%).

\section{REFERENCES}

[1] Dolling, O., Proyecto CICITCA (21/1939): Impacto del reuso del aqua en el proceso de deserification en San Juan y su relacion con la variabilidad climatica. Informe Academico Final (2011-2013), Universidad Nacional de San Juan, Marzo, 2014.

[2] Boukalová, Z. \& Křivánek, Z., Alice-an effective tool of groundwater-level regulation for large vine growing areas. River Basin Management VIII, A Corun̆a, Spain, 2015. ISBN: 978-1-84564-962-3; eISBN: 978-1-84564-963-0.

[3] Boukalova, Z. \& Krivanek, Z., Alert system in case of excess withdrawal or rise up of groundwater in the sensitive areas: the installation of the ALICE system in the vineyard of El Huaico, Pedernal, Argentina. International Journal of Sustainable Development and Planning, 12(1), 2016. ISSN 1743-7601; on-line: ISSN 1743-761X.

[4] http://www.bodegascallia.com/english

[5] PROSAP programme http://www.prosap.gov.ar/py_DescripcionPorJurisdiccion. aspx?j=15 